\title{
Cartographie de l'aléa induit par d'anciennes carrières souterraines en milieu urbain dense
}

\section{S. ARBIZZI}

Laboratoire Régional de l'Est Parisien (LREP) Rue de I'Égalité Prolongée BP 134

93352 Le Bourget Cedex

Sandrine.arbizzi@ equipement.gouv.fr

C. KREZIAK

Laboratoire Régional de I'Est Parisien (LREP) 319 , avenue G. Clemenceau BP 505

77015 Melun Cedex

Charles.kreziak@ equipement.gouv.fr

A.-M.

PRUNIER-LEPARMENTIER

Inspection Générale des Carrières de Paris (IGC)

3, avenue du Colonel Henri Rol-Tanguy 75014 Paris Anne-Marie. Leparmentier@paris.fr
Dans le cadre de l'élaboration du Plan de prévention des risques « Mouvements de terrain » de vingt-deux communes du Val-de-Marne, le LREP et I'IGC de la ville de Paris élaborent de concert les cartes de l'aléa induít par d'anciennes carrières souterraines abandonnées. La cartographie d'aléa repose en particulier sur un travail d'enquête approfondie et le cas échéant sur l'interpolation des zones d'exploitations potentielles. Les études techniques intègrent également des phases de communication qui contribuent à la concertation nécessaire à l'acceptation du projet.

Mots-clés : carrière souterraine, fontis, concertation, interpolation, qualification d'aléa.

\section{Mapping of hazards induced by underground quarries on densely populated urban zones}

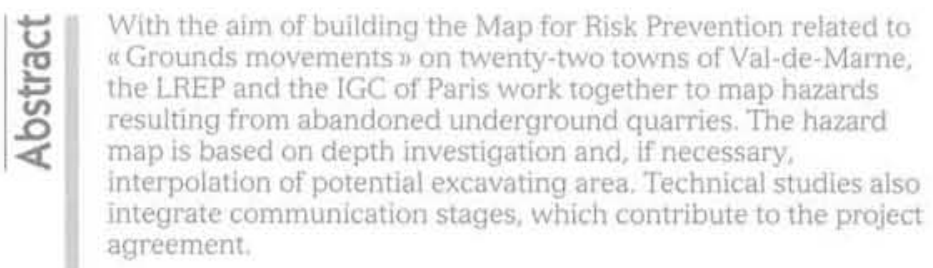

Key words: underground quarry, sinkhole, dialogue,

interpolation, hazard assessment. 


\section{Introduction}

\section{Contexte de prescription du Plan de Prévention des Risques dans le Val-de-Marne}

Du fait des nombreux aménagements en milieu urbain et de la forte mobilité de la population francilienne, les anciennes carrières, autrefois intensivement exploitées pour l'extraction de matériaux, sont progressivement tombées dans l'oubli. Cependant, les différents désordres liés à leur dégradation peuvent brutalement rappeler les risques qu'elles représentent.

Dans les années quatre-vingt, des périmètres d'anciennes carrières souterraines ont été délimités de façon approximative dans le Val-de-Marne pour cerner les zones touchées et soumettre ainsi les permis de construire à l'avis de l'TGC. Environ $96 \%$ des zones sous-minées dans ce département se trouvent en zone urbaine (hors espaces agricoles et espaces naturels) dont $75,4 \%$ sont construites (Leparmentier et al., 2005). Le 1 er août 2001, vingt-deux communes faisaient l'objet d'un arrêté de prescription de Plan de prévention des risques (PPR) « mouvements de terrain », que le service instructeur a souhaité engager au niveau départemental.

Devant l'ampleur de la tâche, la direction départementale de l'Équipement (DDE) a choisi d'étaler les études techniques sur trois ans. Du fait de leurs connaissances locales et de leur expérience en matière d'élaboration de PPR, le Laboratoire régional de l'Est parisien et l'Inspection générale des carrières de la ville de Paris ont été respectivement retenus pour étudier les anciennes carrières de gypse (majoritairement) et les carrières de la proche banlieue.

Les communes ont été regroupées en bassins de risques, établis en fonction de la localisation et de la nature des exploitations (Fig. 1, Tableau I).

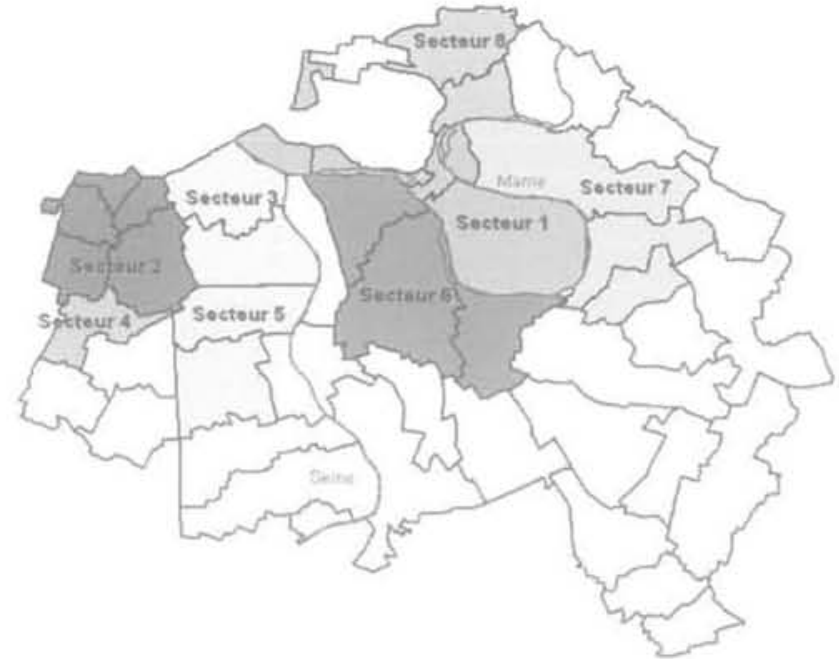

\#G.1 Regroupement des communes concernées par le PPR en huit bassins d'étude.

Municipalities concerned by PPR, grouped in eight risk zones.

\section{2}

\section{Présentation du contexte géologique du Val-de-Marne, des carrières et des principaux désordres}

\section{Contexte géologique et structural du département}

La géologie du Val-de-Marne intéresse la majeure partie des formations de l'ère Tertiaire du bassin parisien, présentée sur le log stratigraphique de la figure 2. Les principaux horizons rencontrés sont les suivants, des plus récents aux plus anciens :

TABLEAu Répartition des bassins à étudier entre l'IGC (secteurs 1-3) et le LREP (4-8), The eight areas shared out between IGC (1-3) and LPEP (4-8) for the study.

\begin{tabular}{|c|c|c|c|c|c|c|c|c|c|}
\hline & \multirow{2}{*}{ Contexte } & \multicolumn{8}{|c|}{ Matériaux exploités (en soutersain ou à ciel ouvert) } \\
\hline & & $\begin{array}{l}\text { Alluvions } \\
\text { anciennes }\end{array}$ & $\begin{array}{c}\text { Limons } \\
\text { des plateaux }\end{array}$ & $\begin{array}{l}\text { Travertin } \\
\text { de Brie }\end{array}$ & Argile verte & Gypse & $\begin{array}{c}\text { Calcaire: } \\
\text { de Champigny }\end{array}$ & $\begin{array}{l}\text { Calcaire } \\
\text { grossjer }\end{array}$ & $\begin{array}{l}\text { Argile } \\
\text { plastique }\end{array}$ \\
\hline 1 & $\begin{array}{c}\text { Boucle de la Marne } \\
\text { à proximité } \\
\text { du bois de Vincennes }\end{array}$ & $\checkmark$ & & & & & & $\checkmark$ & \\
\hline 2 & Vallée de la Bièvre & & $\checkmark$ & $\checkmark$ & $\checkmark$ & $\checkmark$ & & $\checkmark$ & $\checkmark$ \\
\hline 3 & $\begin{array}{c}\text { Terrasse } \\
\text { d'Tvry-sur-Seine }\end{array}$ & $\checkmark$ & & & & & & $\checkmark$ & \\
\hline 4 & $\begin{array}{l}\text { Plateau de Fresnes } \\
\text { et vallée de la Bièvre }\end{array}$ & & & $\checkmark$ & & $\checkmark$ & & & \\
\hline 5 & $\begin{array}{c}\text { Plateau } \\
\text { de Vitry-sur-Seine } \\
\text { et vallée de la Seine }\end{array}$ & & & $\checkmark$ & $\checkmark$ & $\checkmark$ & & & \\
\hline 6 & $\begin{array}{c}\text { Plaine alluviale } \\
\text { entre Seine et Marne }\end{array}$ & $\checkmark$ & & & & $\checkmark$ & & $\checkmark$ & \\
\hline 7 & $\begin{array}{l}\text { Rive gauche } \\
\text { de la Marne }\end{array}$ & & & & & & $\checkmark$ & $\checkmark$ & \\
\hline 8 & $\begin{array}{c}\text { Terminaison } \\
\text { du plateau } \\
\text { de Fontenay-sous-Bois } \\
\text { et vallée de la Marne }\end{array}$ & & & $\checkmark$ & & $\checkmark$ & & & \\
\hline
\end{tabular}


- le calcaire de Brie constitue l'entablement des plateaux. Hétérogène, il présente une alternance de bancs marneux et calcaires assez durs morcelés et parfois meuliérisés (épaisseur $10 \mathrm{~m}$ ) ;

- les argiles vertes sont relativement homogènes, d'une couleur verte caractéristique, plastiques et très peu perméables (épaisseur $-7 \mathrm{~m}$ ). Les marnes à Cyrènes (épaisseur - $1 \mathrm{~m}$ ), sur lesquelles elles reposent, sont argilo-marneuses, feuilletées et fossilifères (Cyrènes) ;

- les marnes supra-gypseuses sont représentées au sommet par des marnes blanches calcareuses : les marnes de Pantin (épaisseur $\sim 4 \mathrm{~m}$ ), puis par des marnes bleues homogènes et compactes : les marnes d'Argenteuil (épaisseur - $10 \mathrm{~m}$ )

- les masses et marnes du gypse et le calcaire de Champigny. L'étage Ludien est marqué dans le Val-de-Marne par une transition entre un faciès calcaire et un faciès gypseux. A l'est du département, une succession de marnes blanches et de calcaire silicifiés détermine le calcaire de Champigny (épaisseur - 15-40 m), tandis qu'au nord-ouest se retrouvent les trois masses de gypse caractéristiques des masses et marnes ;

- les sables de Monceau sont représentés par des sables fins avec une base plus marneuse, généralement en poches (épaisseur $-2 \mathrm{~m}$ ), qui remplissent les surfaces d'érosion du toit des marno-calcaires de Saint-Ouen ; - le marno-calcaire de Saint-Ouen est constituée d'une série de marnes crème à rognons calcaires et de bancs calcaires parfois siliceux, où s'intercalent des feuillets argileux et ponctuellement des niveaux gypseux (épaisseur $-10 \mathrm{~m}$ ) ; - I'horizon de Beauchamp est représenté par des sables quartzeux vert foncé, bleus, verts ou gris, devenant plus argileux à la base (épaisseur $\sim 6 \mathrm{~m}$ ) ;

- les marnes et caillasses sont des marnes blanches plus ou moins argileuses et magnésiennes (épaisseur 10$20 \mathrm{~m}$ ), présentant en partie inférieure des bancs de calcaire siliceux (caillasses). Elles peuvent contenir des épaisseurs non négligeables de gypse albastroïde ;

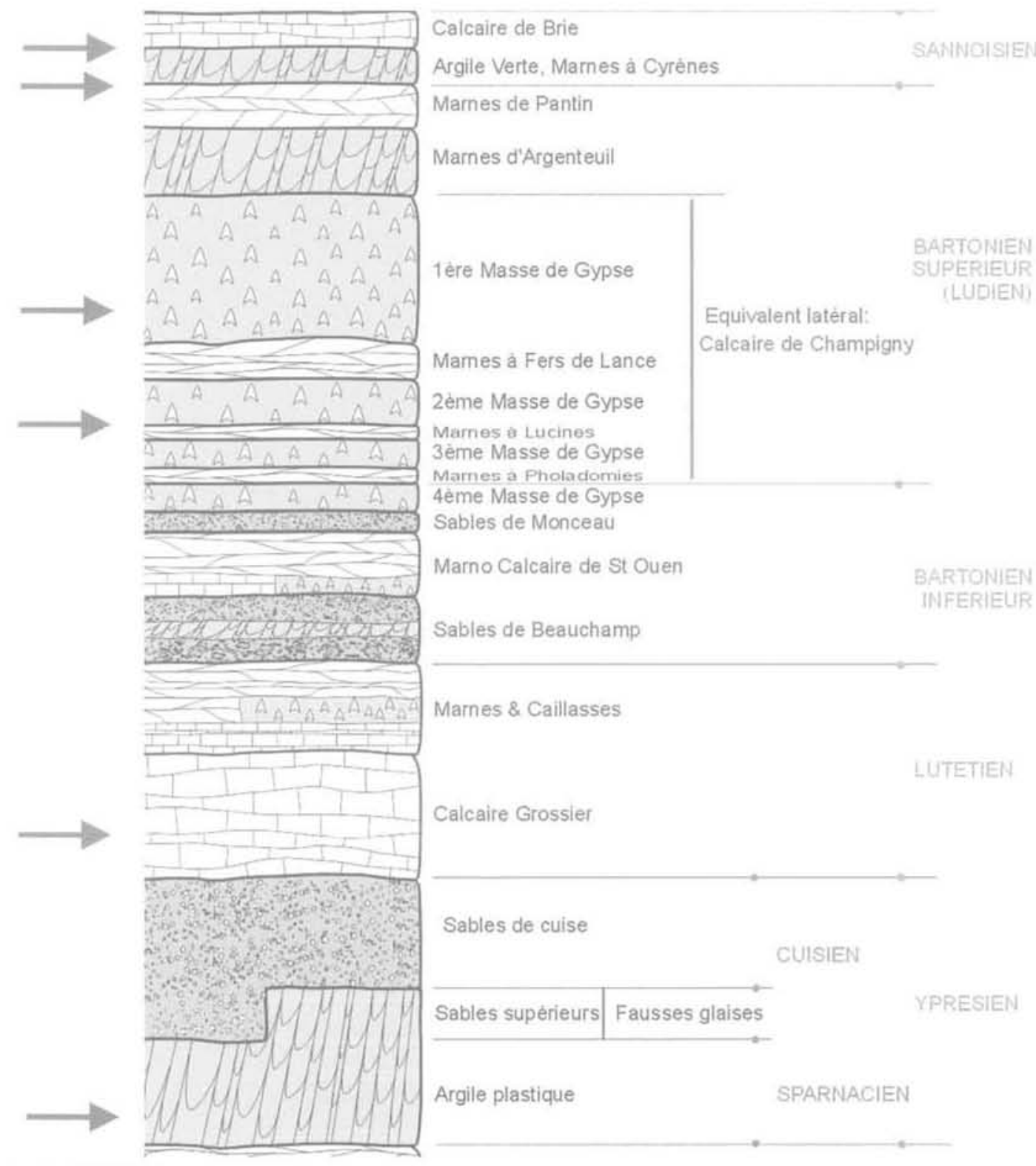

F13.2 Extrait de la coupe stratigraphique des terrains tertiaires de Paris et de ses environs. Les flèches indiquent les principaux matériaux exploités dans le Val-deMarne.

Extract of tertiary soils' stratigraphic column from Paris and surrounding areas. The main extracted soils are highlighted by arrows. 
- le calcaire grossier se divise en trois étages. L'étage supérieur est constitué de bancs lités peu épais alternativement tendres à durs renfermant des cérithes (épaisseur - $10 \mathrm{~m}$ ) ; l'étage moyen regroupe des calcaires grisâtres peu fossilifères à l'exception de milioles (épaisseur - $4 \mathrm{~m}$ ) ; l'étage inférieur est un ensemble sablo-gréseux à cimentations calcaire renfermant des nummulithes (épaisseur $\sim 6 \mathrm{~m}$ ) ;

- les sables cuisiens ne sont pas présents à l'affleurement. Localement, les fausses glaises formées d'argile brune, de lignite et de pyrite, avec des niveaux sableux lenticulaires, peuvent atteindre $5 \mathrm{~m}$;

- l'argile plastique est une masse compacte d'argiles grises, noires, jaunes, lie de vin et panachées à la base (épaisseur jusqu'à $12 \mathrm{~m}$ ).

Ces affleurements tertiaires peuvent être également recouverts par des dépôts postérieurs tels que les dépôts alluvionnaires, les limons des plateaux et les formations de pente :

- dans ce secteur, les allavions sont des dépôts de la Seine, de la Marne et de leurs affluents. Elles sont distinguées en alluvions modernes, matériaux fins à prédominance argilo-sableuse ou limoneuse contenant des matières organiques (épaisseur 1-10 m), et alluvions anciennes, constituées par une alternance de sables et graviers, de cailloutis et de sables fins, leur base étant le plus souvent formée par des dépôts de graviers grossiers (épaisseur jusqu'à $10 \mathrm{~m}$ ). Leur nature peut être différenciée par leur origine (Seine : éléments silico-calcaires avec nombreux silex; marne : dépôts plus sableux et calcaires) ;

- les limons des plateaux ont recouvert au Pléistocène les terrains tertiaires érodés (épaisseur 1-9 m). Ils sont constitués par des dépôts fíns et meubles (action éolienne) de teinte ocre à brun rougeâtre.

Du point de vue structural, le Val-de-Marne est traversé par l'anticlinal de Meudon (Fig. 3), qui donne un pendage important aux assises géologiques à sa proximité, et justifie notamment les facilités d'exploitation du calcaire grossier.

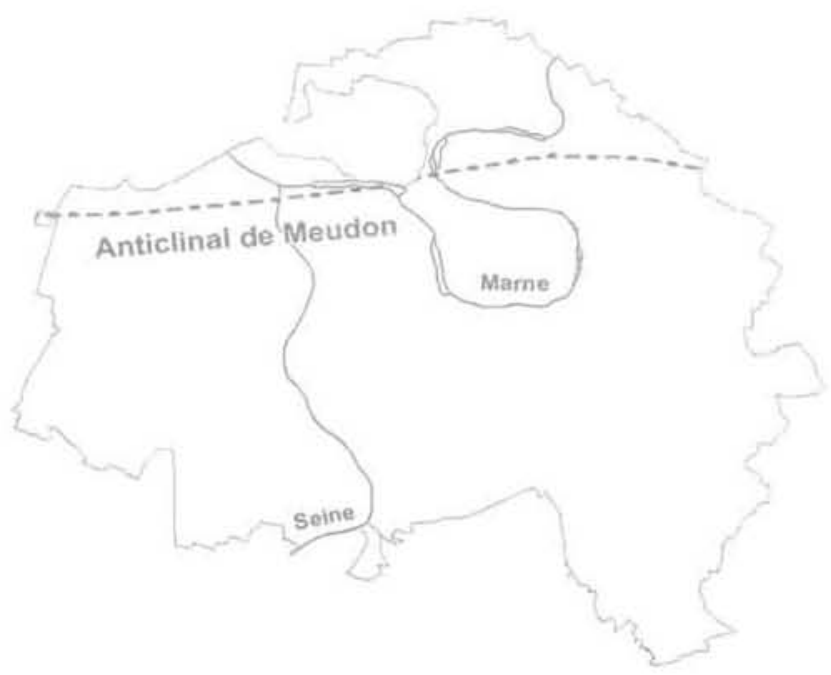

FG.3 L'anticlinal de Meudon au nord du département du Val-de-Marne.

Meudon anticline is localized at the north of the departmental territory.

\section{2}

\section{Historique des carrières}

Les cavités susceptibles d'entrainer des désordres en surface dans le Val-de-Marne concernent principalement le calcaire grossier lutétien (565 ha), le gypse ludien (104 ha, Fig. 4), le calcaire de Champigny ludien et le travertin de Brie sannoisien.

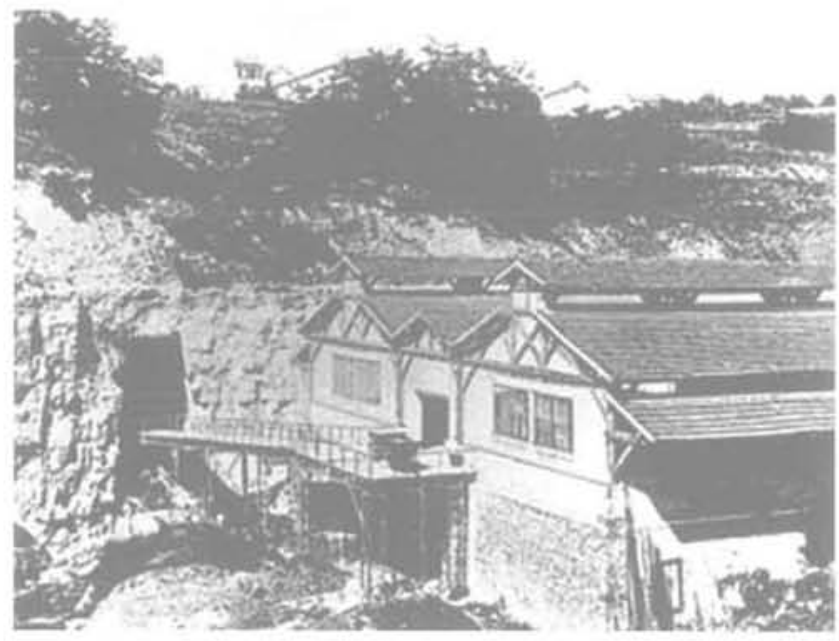

FIG. 4 Carte postale montrant une entrée en cavage vers des galeries souterraines de gypse (ville de Fontenay-sous-Bois). Postcard showing a cave entry to an underground gypsum quarry (City of Fontenaysous-Bois).

Si les plus anciennes exploitations souterraines datent de l'époque gallo-romaine, le développement de carrières plus importantes a, semble-t-il, débuté au IX siècle. Les matériaux calcaires ont principalement été extraits pour fournir de la pierre à bâtir ou produire de la chaux tandis que le gypse était transformé en plâtre.

Ouvertes en périphérie des villes, les cavités ensuite abandonnées se sont rapidement retrouvées sous les faubourgs urbains, avec les conséquences parfois tragiques que la présence de vides importants entraine vis-à-vis de la sécurité des populations. Les accidents répétés et la perte de vies humaines au cours du XVIII ${ }^{*}$ siècle ont conduit à la création à Paris du premier service de contrôle, l'Inspection générale des carrières, par arrèté du conseil d'État du 4 avril 1777 . C'est de cette période que date l'arrêt de toute exploitation sous la ville de Paris intra-muros, même si l'exploitation des carrières souterraines de gypse sous la butte Montmartre s'est prolongée jusqu'à la deuxième moitié du $\mathrm{XIX}$ siècle. En revanche, les extractions se sont intensifiées dans la petite couronne parisienne.

\section{3}

\section{Différentes techniques d'extraction des matériaux}

Les modes d'exploitation se sont succédé et ont varié dans le temps d'un lieu à l'autre, mais les principes généraux sont restés semblables. Ils sont au nombre de quatre : exploitation à ciel ouvert, ou exploítation en souterrain par la méthode des piliers tournés, des " hagues et bourrages » ou des rameaux. A partir 
des affleurements sur les versants, le recouvrement augmentant, les carriers ont extrait en souterrain à partir des entrées en cavage ou des puits d'extraction et de service.

La méthode « des piliers tournés » laisse régulièrement du matériau en place qui constitue des piliers naturels (Figs. 5 et 6). Les salles d'exploitation abandonnées très partiellement remblayées sont hautes (jusqu'à $7 \mathrm{~m}$ ) et présentent de grandes (voire trop grandes) portées. Le taux de défruitement moyen atteint $65 \%$. Cette méthode a été employée pour les carrières de gypse ludien, de calcaire de Champigny et les plus anciennes de calcaire grossier.

La méthode par « hagues et bourrages » consiste à soutenir le ciel de la carrière par des piliers de pierres

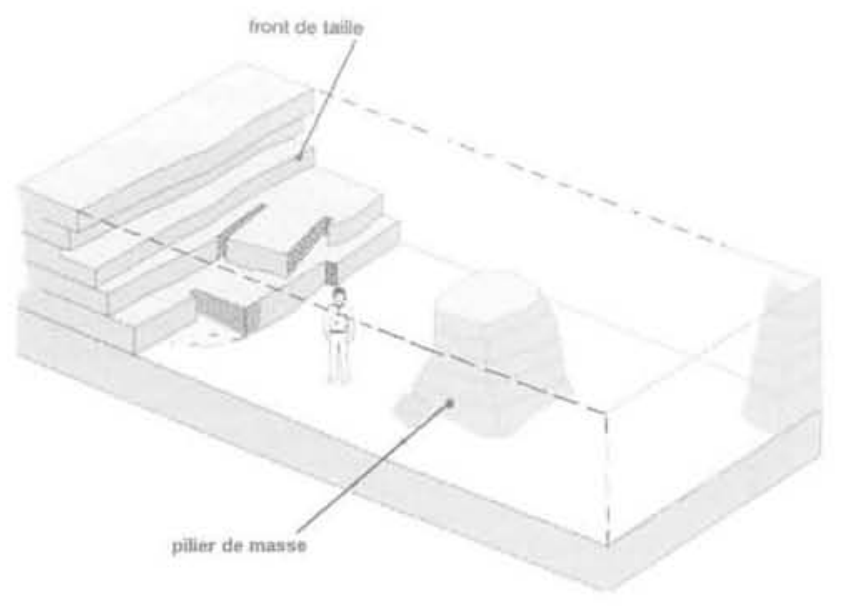

FiG.5 Schéma d'exploitation par piliers tournés. Scherne of a chamber and pillar exploitation.

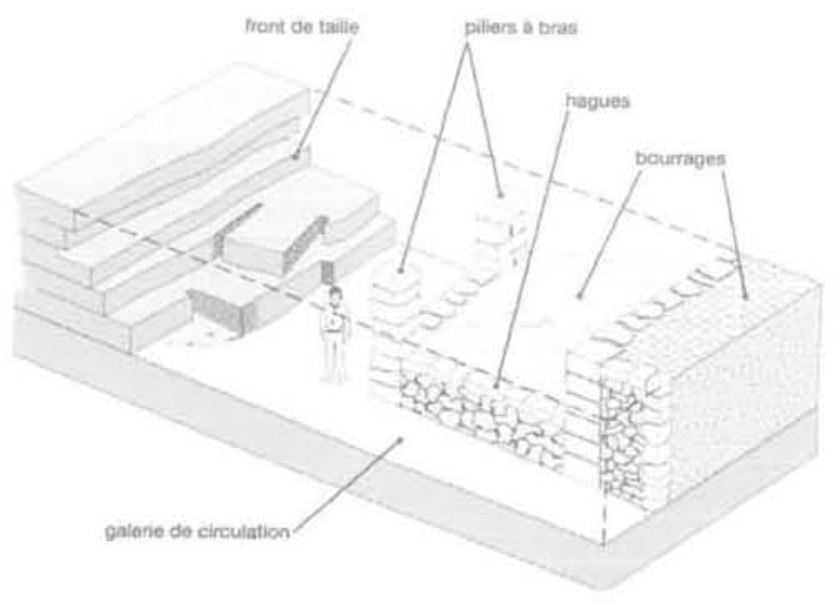

FIG.7 Schéma d'exploitation par hagues et bourrages (IGC).

Scheme of an extraction lead by walls and backfilling. sèches (cales à bras) et à combler les vides par des remblais maintenus par des murs de pierres sèches (hagues) (Figs. 7 et 8). Le taux de défruitement est de $100 \%$ mais les hauteurs de galerie sont moindres (1.5$2 \mathrm{~m}$ en moyenne),

Plus rare, la méthode d'exploitation en rameaux a été employée pour des extractions souvent clandestines, notamment pour certaines couches géologiques peu profondes comme le calcaire de Brie. Elle consiste en une galerie principale ( recherche $n)$ de quelques dizaines de mètres de longueur, complétée par des rameaux perpendiculaires (Fig. 9). Les galeries peuvent être parallélépipédiques ou en voûte. L'accès se faisait par puits et/ou par entrée en cavage depuis le flanc de coteaux ou directement depuis certaines habitations.

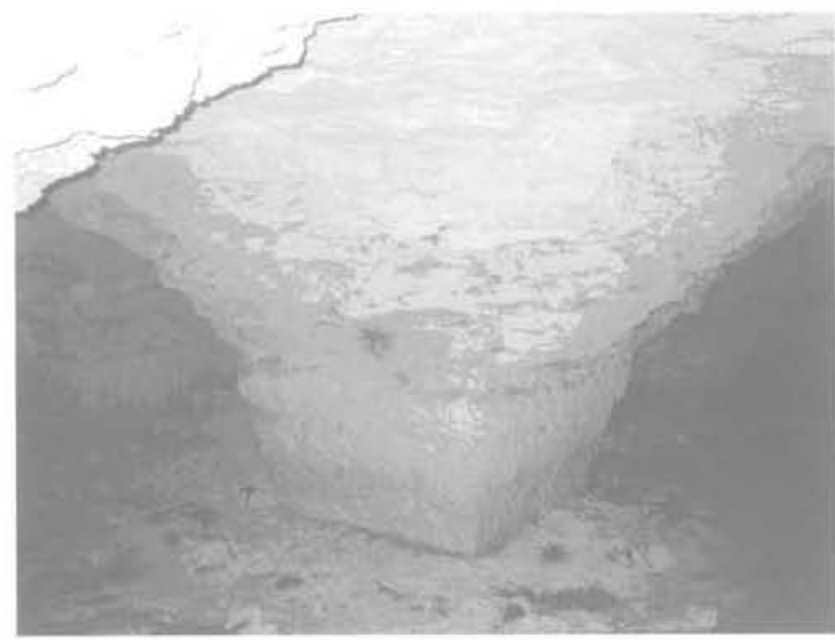

FGG. 6 Pilier tourné dans la masse du calcaire grossier.

Chamber and pillar exploitation in a "calcaire grossier" quarry.

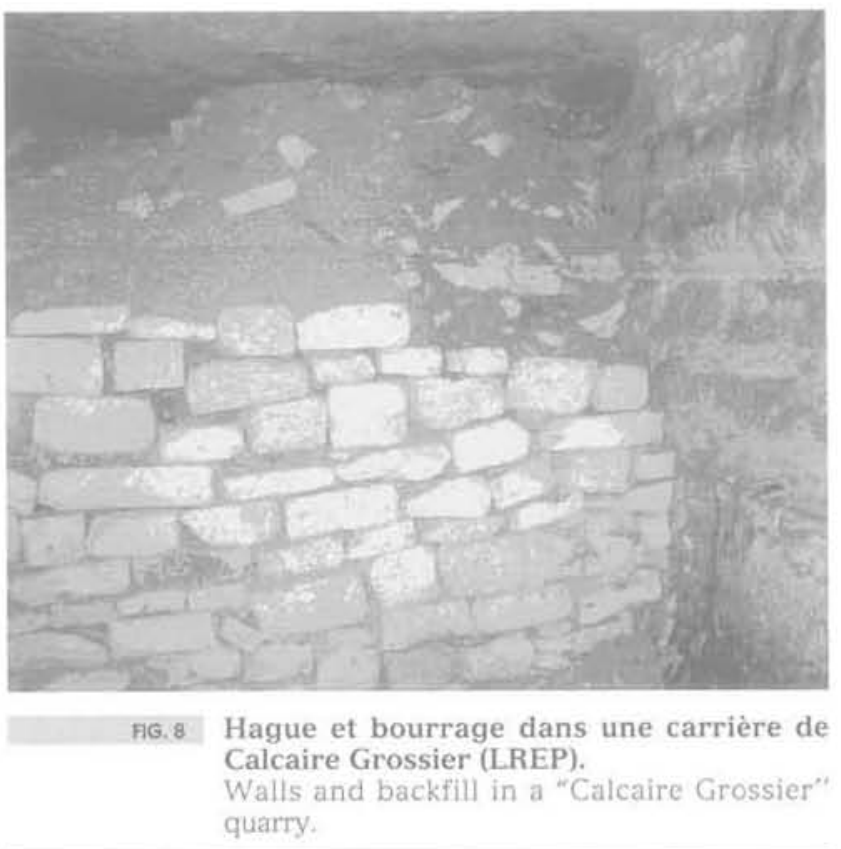




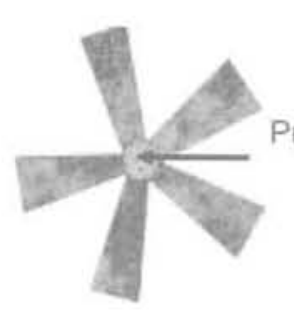

Galeries en étoile
En plan

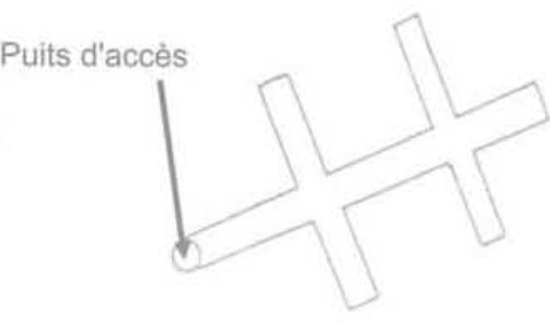

Galeries filantes

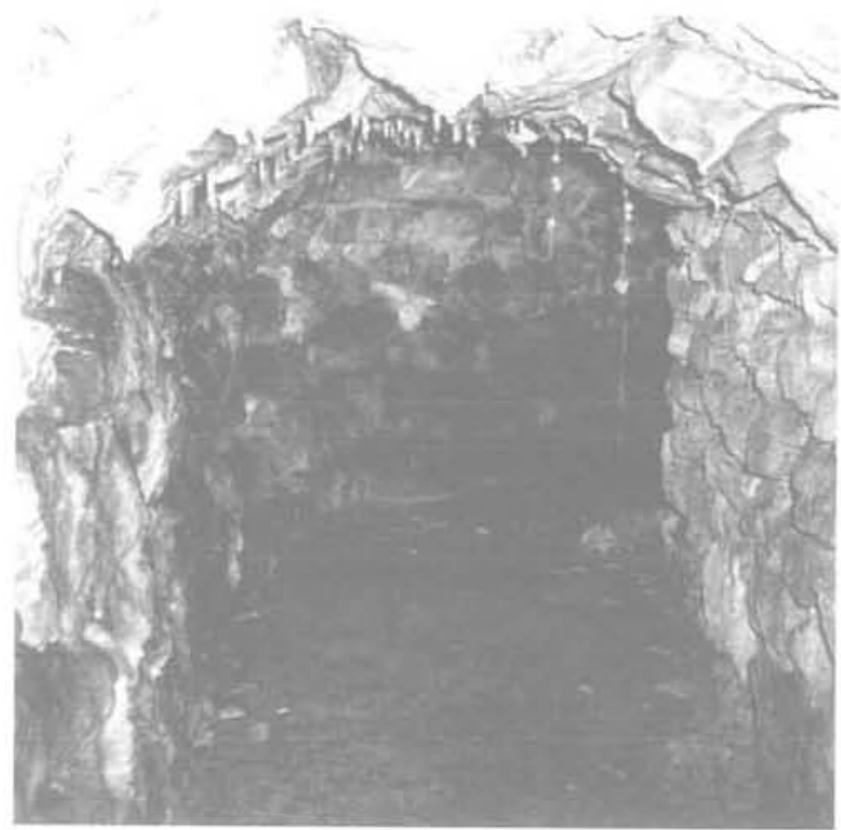

FiG.9 Méthode d'exploitation par galeries en étoiles ou filantes (photographie Tremoureux). Extraction method following a star scheme or narrow gallery (Tremoureux's photography).

\section{Dégradation des anciennes carrières à l'origine de différents désordres en surface}

Dans le cas des exploitations par hagues et bourrages, les galeries de front de taille et de circulation sont restées vides et présentent actuellement des signes visibles de vieillissement tels que l'écaillage, la fragmentation, la fissuration, voire la ruine. Le ciel s'affaisse sur les remblais et casse au front de taille (cisaillement). Les bancs séparatifs entre étages sont souvent très minces (Fig. 10)

Les désordres de surface majoritairement rencontrés pour les autres techniques d'exploitation sont des affaissements ou effondrements. Ils sont consécutifs à la présence de vides dans les bancs de calcaire qui inexorablement vont se refermer, entrainant l'effondrement des terrains sus-jacents. Les vides peuvent remonter vers la surface après affaissement des terrains qui les recouvrent et provoquer alors, selon la hauteur du recouvrement, soit une cuvette plus ou moins importante appelée affaissement soit une cavité appelée fontis (Fig. 11). Les affaissements et les fontis sont des phénomènes localisés, d'une forme circulaire et de diamètre plus ou moins grand. Lorsqu'un effondrement par rupture des piliers tournès concerne une grande partie de la carrière et se fait brutalement, on parle d'un effondrement généralisé.

Les processus de dégradation des carrières résultent souvent d'une combinaison entre une ou plusieurs configurations défavorables susceptibles de modifier les conditions d'équilibre du millieu et d'accélérer la rupture. Les infiltrations d'eau accidentelles (fuite de réseau, absence d'assainissement, cuves non étanches) ou récurrentes (infiltrations de versant, crues, marnages) en sont les principales causes. S'ajoutent les problèmes liés à la géométrie (taux de défruitement trop élevé, murs ou pieds de carrière trop minces, disposition hétérogène des piliers entre étages superposés, décapage des niveaux argileux dans les terrains de recouvrement...) et/ou au couvert végétal (essences à racines abondantes et profondes), qui éclatent les ciels et piliers.
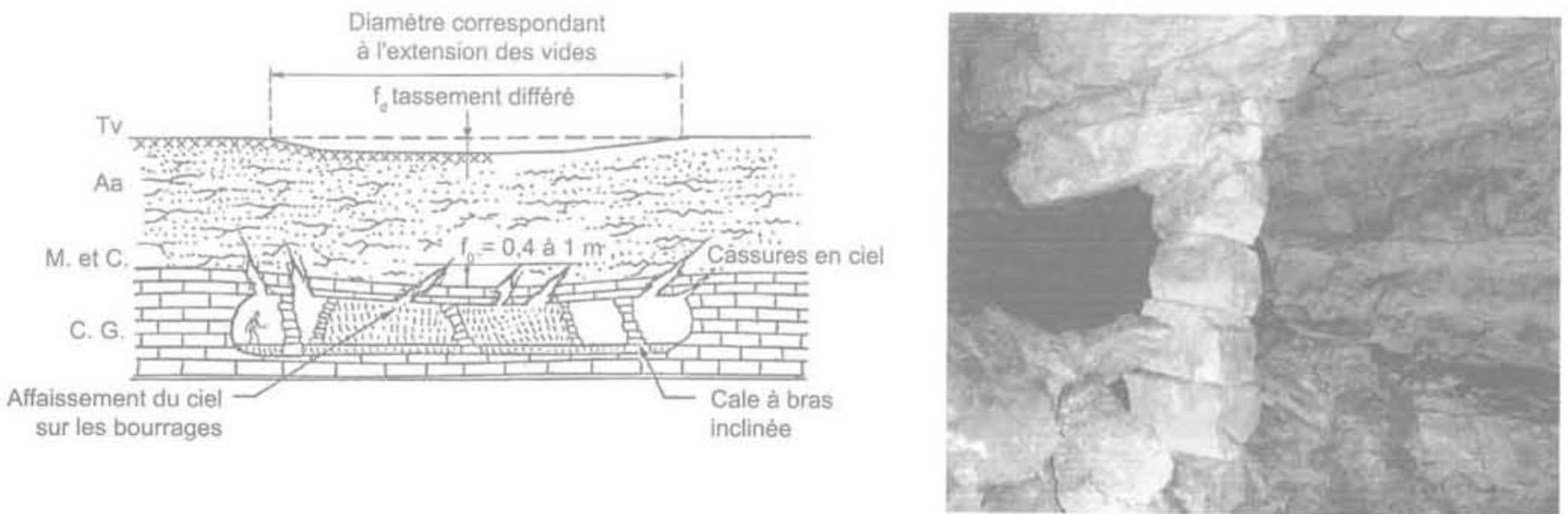

F16: 10 Tassement différé au droit d'une carrière exploitée par hagues et bourrages (IGC). Late settlement above a backfilled quarry (IGC). 


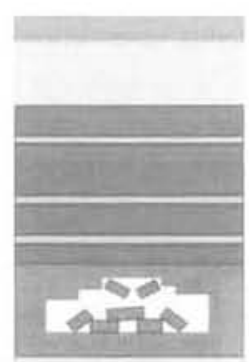

Rupture de toit avec chutes de blocs dans une ancienne exploitation.

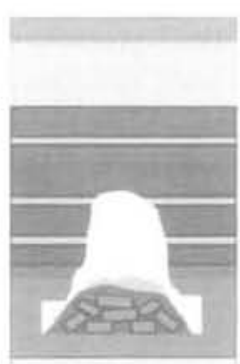

Debbut de formation d'une cloche de fontis.

Un cône đ'éboulis commence à se former.

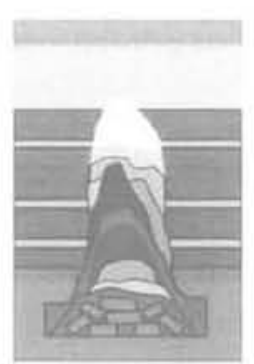

La cloche de fontis continue à se développer vers la surface. Le cóne d'éboulis a remp! la cavité souterraine

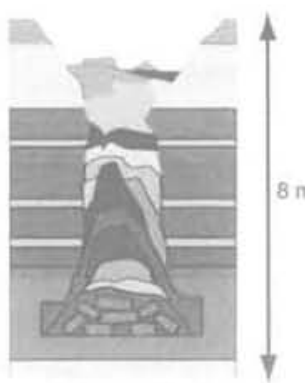

Sulte a Palteration des terrains superficiels le fontis prend une forme d'entonnoir stable.

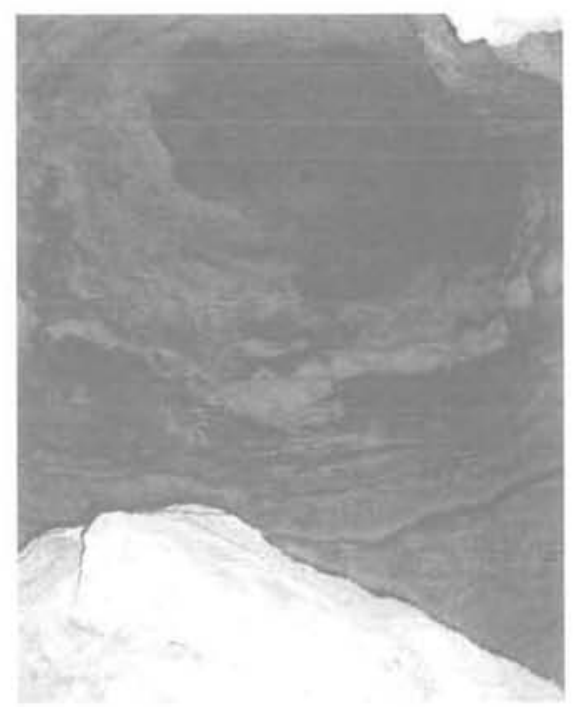

FG.11 Schéma d'évolution d'un fontis (LCPC et al., 2002) et cloche de fontis (IGC). Scheme of a sinkhole evolution (LCPC et al., 2002) and transitory vaulted (IGC).

\section{Conduite des études sur les premiers secteurs étudiés}

\section{1}

\section{Les trois grandes étapes d'élaboration du Plan de prévention des risques}

Le Plan de prévention des risques naturels a été institué par la loi Barnier en 1995, et son élaboration se fait sous la responsabilité et à l'initiative de l'Etat. A travers ses objectifs de connaissance du risque et de réduction de la vulnérabilité, ce document permet de délimiter les zones exposées et de les réglementer en limitant ou conditionnant la constructibilitè. Une fois approuvé, le PPR vaut servitude d'utilité publique et doit être annexé aux documents d'urbanisme. L'élaboration du PPR s'effectue en trois principales étapes (Fig. 12).

La première étape concerne la connaissance du phénomène naturel, qui se traduit principalement par deux cartographies: la carte informative des phénomènes connus, et la carte des aléas. Les études sont menées d'après l'état actuel des connaissances, donc a priori sans campagne d'investigations complémentaires. Cette phase est assurée par le L.REP et l'IGC sur leurs secteurs d'étude respectifs. Un travail d'harmonisation méthodologique a été mené en amont entre les deux organismes afin d'assurer une homogénéitê technique.

\begin{tabular}{|c|c|c|}
\hline $\begin{array}{c}\text { Études } \\
\text { des phénomènes }\end{array}$ & \multirow[t]{2}{*}{$\begin{array}{l}\text { Identification } \\
\text { des enjeux }\end{array}$} & \multirow[t]{2}{*}{$\begin{array}{l}\text { Document } \\
\text { règlementaire }\end{array}$} \\
\hline $\begin{array}{c}\text { Carte informative } \\
\text { des phènomènes naturets }\end{array}$ & & \\
\hline 7 & $\begin{array}{l}\text { Appréciation } \\
\text { des enjeux }\end{array}$ & $\begin{array}{l}\text { Plan de prévention } \\
\text { des risques }\end{array}$ \\
\hline \multicolumn{3}{|l|}{ Carte des aléas } \\
\hline FIG. 18 & \multicolumn{2}{|c|}{$\begin{array}{l}\text { Les trois principales étapes de } \\
\text { l'élaboration du Plan de prévention des } \\
\text { risques. } \\
\text { The three main steps of the elaboration of the } \\
\text { Map for Risk Preventio. }\end{array}$} \\
\hline
\end{tabular}

La deuxième phase consiste à établir une carte des enjeux. Ce document élaboré par la DDE recense les populations, équipements et activités potentiellement concernés par les aléas. L'inventaire porte à la fois sur l'existant mais également sur les projets d'aménagements des collectivités de façon à minimiser les risques à venir. Cette cartographie, croisée avec le contour des zones d'aléa, permet de délimiter les secteurs où des enjeux sont menacés par des aléas, i.e. où il y a des risques (traduction graphique en carte des risques, qui servira de support à la troisième phase de rédaction d'un document réglementaire),

A ce stade de l'étude, cètte phase n'a pas encore été achevée dans les différents secteurs.

\section{2}

\section{Des phases de communication et concertation indispensables}

Le PPR peut être considéré par les collectivitês comme un ensemble de contraintes imposées par l'État et il est indispensable de montrer la convergence des intérêts de chacun vers la prévention des risques et la protection des personnes et des biens.

Avant le lancement des études, le service instructeur adresse aux communes un courrier afin d'introduire les organismes techniques et de faciliter la prise de contact. Une première réunion est organisée en présence de la DDE afin de présenter les objectifs, le déroulement et les conséquences du PPR, permettant d'impliquer les communes et de favoriser la prise de conscience des risques. Divers échanges ont ensuite lieu pour établir un bilan des connaissances des carrières sur le territoire et lister les contacts susceptibles d'apporter des informations intéressantes. La synthèse des renseignements récoltés est ensuite présentée en commune pour validation au regard de la mémoire territoriale. Le point fort de ces échanges est de rassurer sur la transparence de la démarche et surtout d'intégrer les connaissances locales indispensables pour la qualité de l'étude.

Pour l'ensemble des communes du Val-de-Marne concernées par le PPR, le travail d'enquête a notamment été relayé par un article dans la presse locale (Fig. 13).

Ce type de communication contribue pleinement à l'information de la population, indispensable pour 


\section{Val-de-Marnematin}

\section{L'événement}

\section{Les carrières sous surveillance dans 22 communes}

HG. 13 Gros titre du journal Le Parisien du mardi 9 mai 2006 (édition Val-de-Marne).

Banner headlines of "Le Parisien" newspaper. 9th of may 2006 (Val-cie-Marne issue).

l'acceptation du PPR : la première étape pour se prémunir du risque est en effet d'en avoir conscience. De plus, cette communication permet de toucher un public large, qui peut détenir des renseignements insoupconnés sur les carrières. De précieux contacts (associations, particuliers...) ont notamment été obtenus suite à la publication de cet article.

Les premières cartes d'aléas ont étẻ présentées aux communes au cours d'une réunion tripartite rassemblan l'organisme technique, le service instructeur et les ser. vices techniques des communes, dont l'avis est recueilli. La même démarche sera adoptée pour la carte des enjeux.

Bien que cette démarche ouverte sur les échanges et la concertation puisse paraitre lourde, elle est nécessaire pour l'appropriation des conclusions de l'étude et des prescriptions qui seront induites par le PPR. Elle s'inscrit dans les recommandations du guide de la concertation (MEDD, 2002).

\section{Un important travail de synthèse des informations relatives au territoire étudié}

Le travail du LREP et de l'IGC consiste à rassembler tous les renseignements disponibles sur le contexte géomorphologique, géologique et hydrologique de chaque commune étudiée ainsi que sur l'historique des carrières, leurs caractéristiques et leur état actuel. Dans cette étude, l'objectif du PPR est d'affiner les limites des périmètres grossiers (datant des années quatre-vingt) et de qualifier les niveaux d'aléas dans chaque zone exposée. Les anciennes exploitations à ciel ouvert, non prises en compte dans les périmètres antérieurs, sont également considérées.

Les principaux éléments sont puisès dans les archives internes de I'TGC et du LREP, les premières fournissant des cartes des carrières connues au $1 / 1000$ et $1 / 20000$ et des écorchés géologiques, les secondes des reconnaissances géotechniques, études géologiques et hydrologiques ou expertises. Dans le cas de carrières encore accessibles, l'TGC effectue également des visites. La quantité d'informations détenues est relativement importante, du fait de l'ancienneté de l'activité de l'IGC et du grand nombre d'aménagements auxquels le LREP a participé.

Les observations de terrain sont rendues difficiles par la densité du tissu urbain, mais quelques désordres sur bâtis peuvent être constatés. L'analyse est complétée par une photo-interprétation en stéréoscopie. Sur le premier secteur étudié, le LREP a examiné des clichés allant des années 1926 à 1957, à des échelles comprises entre $1 / 20000$ et $1 / 5000$. En dehors des désordres débouchant en surface, ces photographies permettent d'observer les aménagements associés aux carrières (entrées en cavage, puits de ventilation comme indiqué sur la figure 14), Ces éléments indiquent des extensions non cartographiées et des zones où des terrassements ont pu faire disparaître les anciennes galeries et où il n'existe plus de vides souterrains significatifs.

Les acteurs présents sur le territoire, notamment les différents concessionnaires, sont sollicités par courrier pour leur connaissance du sous-sol et des consolidations effectuées. Cette collecte est doublée d'enquêtes de terrain, dans lesquelles sont associés les services techniques des communes, le conseil général, les asso-

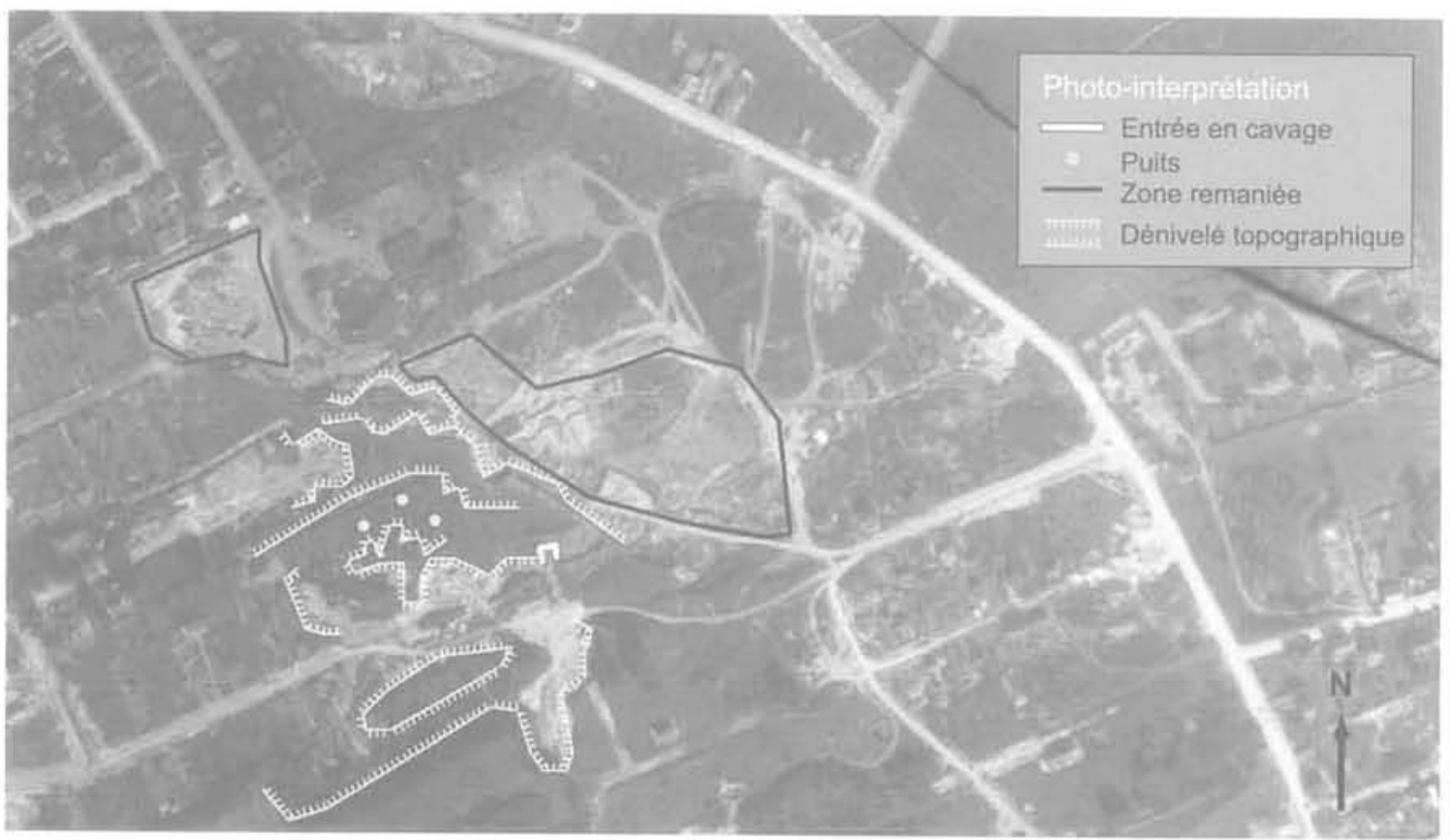

FG.14 Cliché aérien (1926) au droit d'exploitations de calcaire grossier sur les communes de Maisons-Alfort et Créteil (LREP, 2005).

Aerial photograph (1926) over a "calcaire grossier" minig area on Maisons-Alfort and Créteil territories (LREP, 2005). 
ciations, des historiens locaux, des $"$ anciens $x$ ayant une mémoire locale, la RATP, certaines entreprises de BTP. Les archives départementales et celles de la DRIRE sont également consultées.

La synthèse des données récoltées est enfin représentée sur la carte informative qui reflète l'ètat des connaissances à la date de la conduite de l'étude (rendu à l'échelle $1 / 5000$ ). La figure 15 en présente un extrait sur les communes de Maisons-Alfort et Créteil.

\section{4}

\section{Des extensions de carrières à interpréter}

Les cartes détenues par l'IGC sont riches en informations mais peuvent se révéler localement déficientes, celles-ci étant issues de plans parfois anciens et imprécis. Les indices de galeries, hors périmètre sous-miné connu, confirment l'hypothèse que les exploitations aient pu se prolonger. Toutefois, l'interprétation de l'extension possible des zones exploitées est difficile à déterminer pour des carrières inaccessibles, et les études dans le cadre du PPR ne peuvent s'appuyer sur des reconnaissances complémentaires. Sur le premier secteur étudié par le LREP, l'interprétation de l'extension des carrières a été appréhendée par une modélisation numérique des zones potentiellement exploitables et d'après d'anciennes limites cadastrales.

\section{4}

\section{La limite d'exploitabilité du calcaire grossier}

Sur les communes de Créteil et Maisons-Alfort, le toit de la couche géologique a été modélisé (Fig. 16) à partir des sondages pertinents disponibles (données LREP, BSS et IGC, soit 564 sondages).

Au vu des dates anciennes des exploitations connues, il a été considéré que l'extraction n'avait été menée que lorsque le matériau se trouvait hors nappe, l'exhaure étant techniquement et économiquement peu probable. La comparaison de la cote du calcaire grossier et du niveau de nappe (prise à 29 m NGF d'après les chroniques en notre possession) a alors permis de délimiter un périmètre d'exploitabilité (Fig. 17). Dans la partie sud de la zone, le calcaire èmergé décrit une ondulation (influence de l'anticlinal de Meudon) qui coincide avec la répartition des carrières connues. Cette modélisation a ainsi permis de valider ou d'écarter certains indices (Fig. 18).

Il convient de souligner qu'à quelques exceptions près toutes les exploitations connues s'inscrivent dans le périmètre identifié. Les exceptions constatées se situent dans des secteurs où peu de sondages sont disponibles, i.e. où la modélisation est donc moins pertinente.

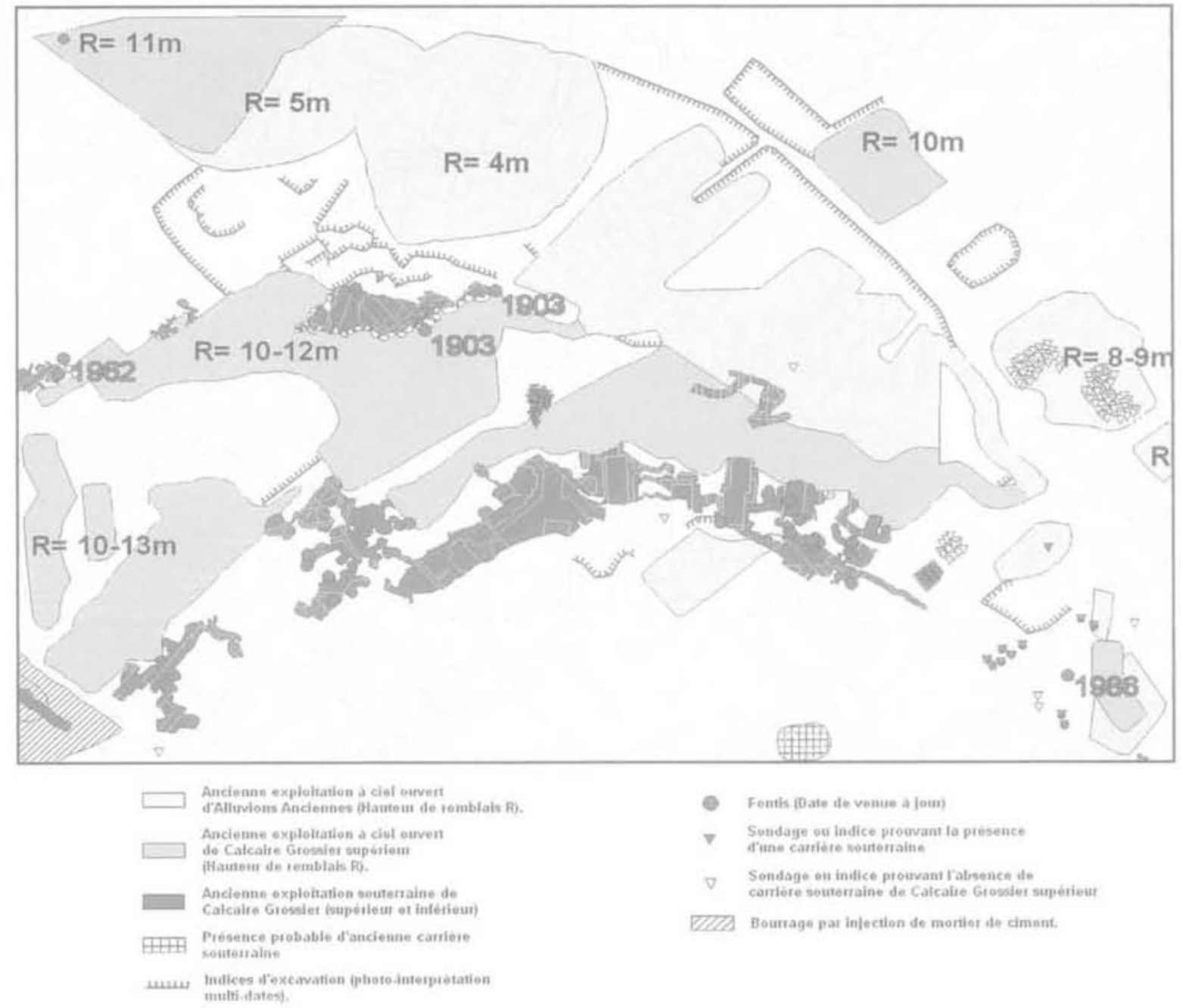

FIG.15 Extrait de carte informative : phénomènes historiques ou observés (LREP, 2005). Extract from the "informative" map: historical or observed phenomena (LREP, 2005). 


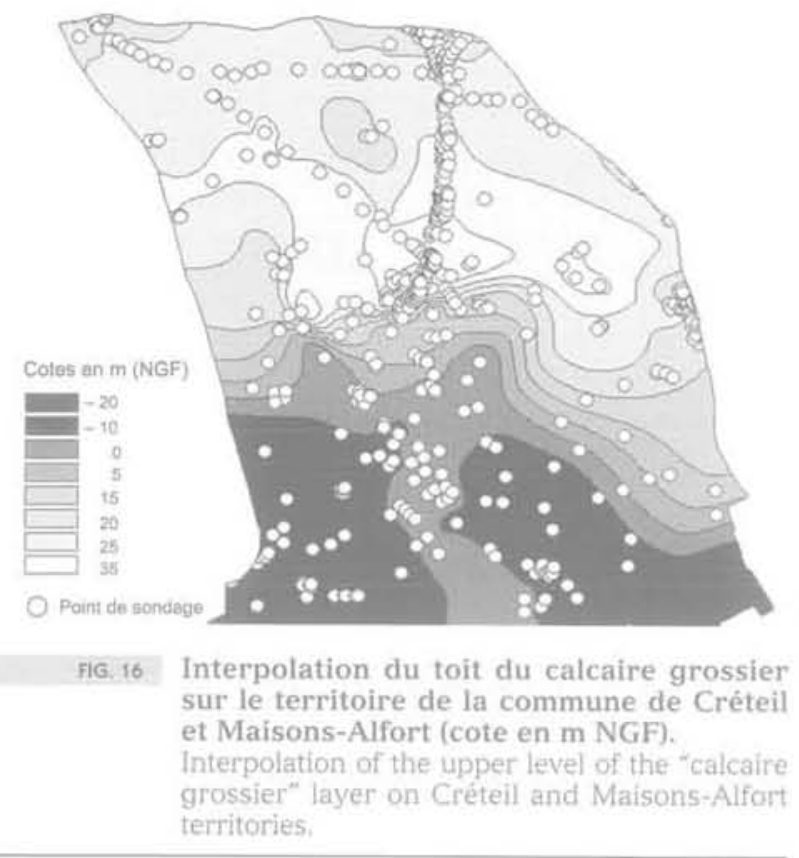

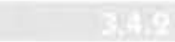

\section{Les anciennes limites cadastrales}

Dans les secteurs où les informations précédentes se révélaient insuffisantes pour conclure à l'extension plausible de certaines carrières, le cadastre napoléonien a servi de repère (cartes de 1813-14 et 1841). Il a en effet été considéré que les extensions pouvaient être approchées au travers des parcelles traversées par des galeries. Si tel est le cas, des droits d'exploitations y ont été acquis, et des extensions ont pu être creusées sur toute la superficie ; toute la parcelle est donc concernée,

De plus, il a été supposé que l'exploitation souterraine ait pu se poursuivre sous la voirie malgré les interdictions. En ce qui concerne les exploitations à ciel ouvert, il a en effet été retrouvé plusieurs anciens documents faisant état d'infractions : l'extraction était parfois frauduleusement menée à moins de $10 \mathrm{~m}$ des chemins vicinaux.

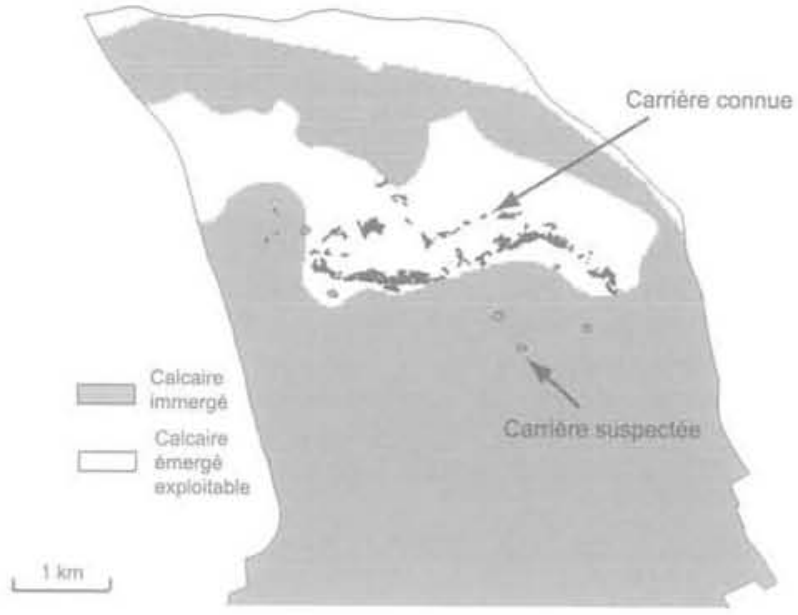

AG. 17 Cartographie des zones de calcaire émergé.

Map of over water limestone.

\section{Qualification des aléas liés aux anciennes carrières}

Les aléas liés aux carrières dépendent non seulement de la géométrie générale (profondeur, type d'exploitation, qualité du remblaiement, protection du front de taille contre les infiltrations d'eau, qualité du recouvrement...), mais aussi du contexte géographique à l'origine de facteurs aggravants (urbanisation, proximité d'un versant, végétation....). Au vu des caractéristiques des anciennes carrières du Val-de-Marne, le risque de fontis et/ou d'affaissement est élevé dans toutes les zones concernées par les anciennes extractions souterraines vides ou partiellement remblayées.

L'intensité de l'aléa est définie en fonction de l'impact estimé sur les personnes et sur les biens : risque d'atteinte à la vie de personnes, fissurations plus

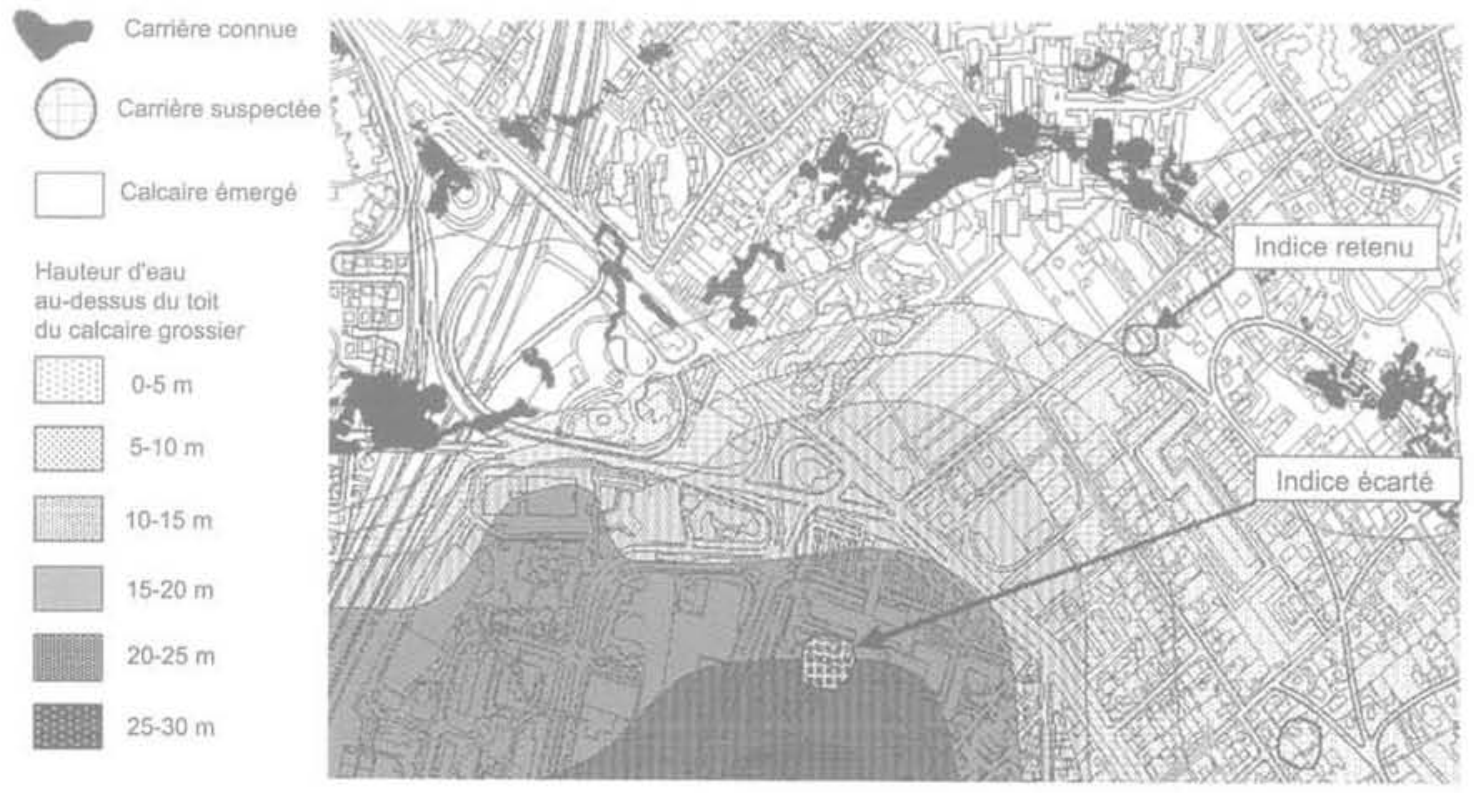

FG. 18 Report des carrières et indices sur la carte des calcaires hors nappe.

Quarries and suspected zones on over water limestone map. 
TABLEAUX\|F⿻ Niti Neaux d'aléas et valeurs des bandes de sécuritê (ZP, MR) retenues. Hazard levels and values of adopted security zones (ZP, MR)

\begin{tabular}{l|c}
\multicolumn{1}{c|}{ Gontexte } & Aléa retenu \\
\hline Fontis repèré & Très fort \\
\hline $\begin{array}{l}\text { Galeries vides/partiellement } \\
\text { remblayées d'origine }\end{array}$ & Fort \\
\hline Galeries uremblayées " & Modéré \\
\hline $\begin{array}{l}\text { Galeries «remblayées " } \\
\text { clavées }\end{array}$ & Modéré \\
\hline Galeries « consolicées » & Faible \\
\hline Puits d'accès non ceinturés & Fort à faible \\
\hline
\end{tabular}

\begin{tabular}{c|c|c} 
Contexte & ZP & MR \\
\hline Carriêres « consolidées v et entrée en cavage & 0 & 0 \\
\hline Carrières « remblayées » & $2 \mathrm{~m}$ & $4 \mathrm{~m}$ \\
\hline Carrières vides de hauteur $<4 \mathrm{~m}$ & $4 \mathrm{~m}$ & $8 \mathrm{~m}$ \\
\hline Carrières de hauteur $>4 \mathrm{~m}$ ou à 2 étages & $8 \mathrm{~m}$ & $16 \mathrm{~m}$ \\
\hline
\end{tabular}

ou moins importantes du bâti, voire mise en péríl ou ruine de la structure. Il est nécessaire d'apprécier également la probabilité d'occurrence. Contrairement à certains aléas engendrés par d'autres phénomènes naturels, ceux liés aux anciennes carrières, tels le fontis et l'effondrement généralisé, se produisent a priori une seule fois et ne peuvent être appréhendés par leur période de retour.

A partir de ces critères quatre niveaux d'aléas ont été déclinés et cartographiés au 1/10 000. Les confortations réalisées ont également été considérées (Tableau II).

Une zone de protection et une marge de reculement ont été retenues (Fig. 19) : la première d'un même niveau d'aléa que les zones sous-minées, la seconde d'un niveau inférieur. La zone de protection (ZP) cor-
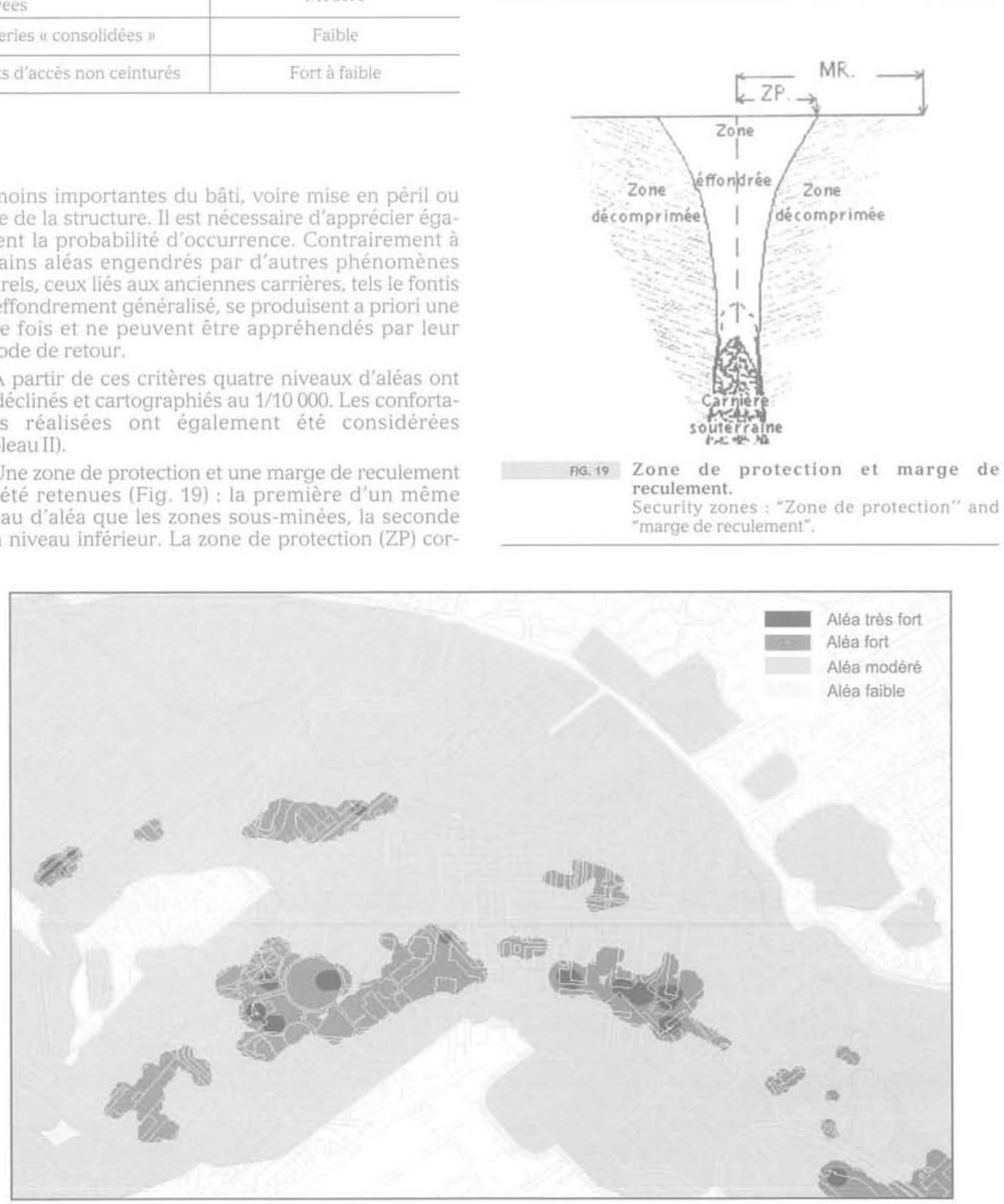

FG. 20 La figure 20 présente un extrait de la cartographie obtenue.

Extrait de carte d'aléas (LREP, 2005).

Extract of the hazard map (LREP, 2005). 
respond à la bande de terrain, susceptible de s'effondrer, lors la survenue d'un fontis en surface ou peu de temps après. La marge de reculement (MR) représente la zone d'influence d'un événement survenu en surface. Au-delà, aucun désordre n'est à craindre en surface. Ces bandes dépendent du matériau exploité, du type d'exploitation et de la présence éventuelle d'exploitations superposées.

Les distances retenues pour les zones de sécurité sont reportées dans le tableau III. Elles ont été déterminées d'après le retour d'expérience de l'TGC sur les désordres survenus dans le Val-de-Marne (environ 140 recensés) : les fontis au droit de carrières de calcaire grossier viennent généralement à jour avec un diamètre de l'ordre de 2 à $3 \mathrm{~m}$.

La figure 20 présente un extrait de la cartographie obtenue.

\section{5}

\section{Esquisse des prescriptions réglementaires}

Bien que les études ne soient pas encore finalisées, il est possible, au regard des pratiques régionales, d'énoncer le type de mesures réglementaires susceptibles d'être retenues.

Dans les secteurs oủ un risque très fort existe, l'urbanisation est généralement figée par une inconstructibilité. Seuls les travaux liés aux infrastructures indispensables à la continuité du service public sont admis. Dans les zones moyennement ou faiblement exposées, la définition des dispositions constructives est subordonnée aux conclusions d'une étude géotechnique préalable. Suivant le niveau du risque et la nature de l'infrastructure (établissement recevant du public ou bâti privé), cette étude est imposée ou recommandée.
En parallèle, un certain nombre de dispositions préventives et de sauvegarde sont établies (diagnostic régulier des réseaux d'assainissements, délimitation de zone de protection en cas d'effondrement...).

\section{6}

\section{Conclusion}

L'élaboration de la cartographie d'aléa sur les communes des premiers bassins de risque nous conduit à retenir quelques points importants, du point de vue méthodologique :

- les secteurs en milieu urbain bénéficient de nombreuses études qui facilitent la compréhension du territoire, Il s'agit d'un véritable avantage étant donné les délais et les moyens associés à une étude de type PPR:

- la dispersion des données techniques représente cependant un frein important à l'avancée de l'étude. Les maîtres d'œuvre et plus encore les maîtres d'ouvrages n'accordent que peu d'importance à la pérennité des archives. De fait, il convient de recouper soigneusement toutes les sources d'informations pour éviter une synthèse incomplète et donc erronée :

- la concertation avec les acteurs locaux est un gage de qualité et d'acceptation de l'étude. Les informations récoltées apportent une richesse indispensable au travail de synthèse. Les échanges permettent en outre une prise de conscience du risque et favorisent l'appropriation du projet ;

- enfin, il est à souligner que la pression foncière en milieu urbain peut être telle que les prescriptions réglementaires peuvent en être influencées. A titre d'exemple, la réalisation de travaux a priori lourds peut être envisagée dans les projets de construction, au vu du niveau de prix des terrains à bâtir dans la région.

\section{Bibliographie}

\footnotetext{
« Fontenay Notre Ville n-Balade au temps passé : collection de cartes postales anciennes de Fontenay-sous-Bois.

Le Parisien du 9 mai 2006. Première page de l'édition Val-de-Marne matin.

LCPC. INERIS, MATE - Évaluation des aléas liés aux cavités souterraines. Guide technique. Collection a Environnement - Les risques naturels $3,2002$.
}

LREP - Plan de prévention des risques naturels. Cartographie de l'aléa mouvement de terrain. Département du Val-de-Marne. communes de Créteil. Maisons-Alfort. Bonneuil-sur-Marne, Dossier 2.1.17049, 2005.

Ministère de l'Écologie et du Développement durable - Plans de prévention des risques naturels. Guide de la concertation. La documentation francaise, 2002.
Prunier-Leparmentier A.M. Delannoy M.A., Lebrun E. - Les PPR mouvements de terrain : les interventions de l'TGC. Les cahiers de I'TAURIF $n^{\circ} 142,2005$. p. 75-85. 